Lifetime measurements and transition probabilities in Mo II

This article has been downloaded from IOPscience. Please scroll down to see the full text article.

2010 J. Phys. B: At. Mol. Opt. Phys. 43085004

(http://iopscience.iop.org/0953-4075/43/8/085004)

The Table of Contents and more related content is available

Download details:

IP Address: 193.190.193.2

The article was downloaded on 07/04/2010 at 08:46

Please note that terms and conditions apply. 


\title{
Lifetime measurements and transition probabilities in Mo II
}

\author{
H Lundberg ${ }^{1}$, L Engström ${ }^{1}$, H Hartman ${ }^{2}$, H Nilsson ${ }^{2}$, P Palmeri ${ }^{3}$, \\ P Quinet $^{3,4}$ and É Biémont ${ }^{3,4}$ \\ ${ }^{1}$ Department of Physics, Lund University, PO Box 118, SE-221 00 Lund, Sweden \\ ${ }^{2}$ Lund Observatory, Lund University, PO Box 43, SE-221 00 Lund, Sweden \\ ${ }^{3}$ Astrophysique et Spectroscopie, Université de Mons-UMONS, 20 Place du Parc, B-7000 Mons, \\ Belgium \\ ${ }^{4}$ IPNAS, Université de Liège, B15 Sart Tilman, B-4000 Liège, Belgium \\ E-mail: E.Biemont@ulg.ac.be
}

Received 4 February 2010, in final form 11 March 2010

Published 5 April 2010

Online at stacks.iop.org/JPhysB/43/085004

\begin{abstract}
Lifetimes have been measured using time-resolved laser-induced fluorescence for 16 odd levels in the doublet, quartet and sextet systems of Mo II, with energies in the range 48000 $61000 \mathrm{~cm}^{-1}$. Absolute transition probabilities and oscillator strengths are determined for $110 \mathrm{UV}$ and visible transitions $(208<\lambda<485 \mathrm{~nm})$ from a combination of experimental lifetimes and theoretical branching fractions. The theoretical results are obtained using the HFR method including core polarization effects.
\end{abstract}

\section{Introduction}

Accurate atomic transition probabilities for heavy atoms and ions are needed in different fields of physics, including astrophysics and fusion research. An efficient and wellestablished approach for obtaining the required data consists of combining lifetime measurements using selective laser excitation with theoretical or experimental branching fraction (BF) determinations. For experimental BFs Fourier transform spectroscopy (FTS) is an appropriate and efficient method.

Experimental transition probabilities in Mo II are scarce. The first results were the arc measurements of Corliss and Bozman (1962), but these results are now known to be affected by large systematic errors and they have been superseded by more recent data. Schnehage et al (1983) determined relative $f$-values for 174 Mo I and 58 Mo II transitions in the range $24.7-557.0 \mathrm{~nm}$ from wall-stabilized arc and hollow cathode measurements. The Mo I data were normalized to an absolute scale using four experimental lifetime values from Kwiatkowski et al (1981). However, the oscillator strengths for Mo II were reported on a relative scale only as no Mo II lifetimes were available at that time.

The first lifetime measurements in Mo II are due to Hannaford and Lowe (1983). They measured lifetimes for 15 levels of the $\mathrm{z}^{6} \mathrm{~F}^{\circ}, \mathrm{z}{ }^{4} \mathrm{P}^{\circ}, \mathrm{z}^{6} \mathrm{P}^{\circ}$ and $\mathrm{z}^{6} \mathrm{D}^{\circ}$ terms using the technique of laser-induced fluorescence (LIF) from a sputtered metal vapour.

Experimental BFs in Mo II for transitions from 197 to $437 \mathrm{~nm}$ have been measured by Sikström et al (2001) from intensity-calibrated spectra recorded with the Lund UV Fourier transform spectrometer. This paper also reported radiative lifetimes for ten levels measured using LIF. Oscillator strengths of 91 transitions were derived by combining the BFs with all available experimental lifetimes.

Theoretical lifetimes and oscillator strengths in Mo II have been calculated by Quinet (2002) using a relativistic Hartree-Fock (HFR) approximation including corepolarization (CPOL) effects. An extensive set of new $f$-values was calculated for 386 Mo II lines in the wavelength range extending from 180 to $746 \mathrm{~nm}$.

The lifetimes measured by Hannaford and Lowe (1983) concern levels with energies in the range 45800$50700 \mathrm{~cm}^{-1}$. Lifetimes for higher energy levels are missing although transitions from these levels are likely to be observed in astrophysics or in plasma physics. The purpose of the present paper is to extend the measurements of Hannaford and Lowe (1983) to levels of higher energies. Thus, we report lifetime measurements for levels with energies in the range $48000-61000 \mathrm{~cm}^{-1}$. Transition probabilities are also deduced for $110 \mathrm{UV}$ and visible transitions depopulating the levels for which new lifetime values have been obtained. 
Table 1. Mo II levels considered and the corresponding excitation schemes.

\begin{tabular}{lllll}
\hline Energy $^{\mathrm{a}}\left(\mathrm{cm}^{-1}\right)$ & Designation $^{\mathrm{a}}$ & Excitation $\lambda_{\text {air }}(\mathrm{nm})$ & Laser mode & Observed $\lambda_{\text {air }}(\mathrm{nm})$ \\
\hline 48022.815 & $4 \mathrm{~d}^{4}\left({ }^{5} \mathrm{D}\right) 5 \mathrm{p} \mathrm{z}^{4} \mathrm{P}_{3 / 2}^{\circ}$ & 208.17 & $3 \omega$ & $280.8,324.1$ \\
50302.913 & $4 \mathrm{~d}^{4}\left({ }^{5} \mathrm{D}\right) 5 \mathrm{p} \mathrm{z}^{6} \mathrm{D}_{7 / 2}^{\circ}$ & 263.88 & $3 \omega+2 \mathrm{~S}$ & 267.3 \\
51373.170 & $4 \mathrm{~d}^{4}\left({ }^{5} \mathrm{D}\right) 5 \mathrm{p} \mathrm{z}^{4} \mathrm{~F}_{3 / 2}^{\circ}$ & 256.63 & $3 \omega+2 \mathrm{~S}$ & 370.3 \\
51732.690 & $4 \mathrm{~d}^{4}\left({ }^{5} \mathrm{D}\right) 5 \mathrm{p} \mathrm{z}^{4} \mathrm{~F}_{5 / 2}^{\circ}$ & 257.44 & $3 \omega+2 \mathrm{~S}$ & $274.6,369.3$ \\
52217.587 & $4 \mathrm{~d}^{4}\left({ }^{5} \mathrm{D}\right) 5 \mathrm{p} \mathrm{z}^{4} \mathrm{~F}_{7 / 2}^{\circ}$ & 257.94 & $3 \omega+2 \mathrm{~S}$ & $271.7,368.8$ \\
52843.296 & $4 \mathrm{~d}^{4}\left({ }^{5} \mathrm{D}\right) 5 \mathrm{p} \mathrm{z}^{4} \mathrm{~F}_{9 / 2}^{\circ}$ & 253.85 & $3 \omega+2 \mathrm{~S}$ & $267.3,278.5,363.5$ \\
54238.949 & $4 \mathrm{~d}^{4}\left({ }^{5} \mathrm{D}\right) 5 \mathrm{p} \mathrm{z}^{4} \mathrm{D}_{1 / 2}^{\circ}$ & 235.45 & $3 \omega+\mathrm{S}$ & $334.7,338.0$ \\
54688.002 & $4 \mathrm{~d}^{4}\left({ }^{5} \mathrm{D}\right) 5 \mathrm{p} \mathrm{z}^{4} \mathrm{D}_{3 / 2}^{\circ}$ & 234.38 & $3 \omega+\mathrm{S}$ & 329.8 \\
55216.203 & $4 \mathrm{~d}^{4}\left({ }^{5} \mathrm{D}\right) 5 \mathrm{p} \mathrm{z}^{4} \mathrm{D}_{5 / 2}^{\circ}$ & 231.51 & $3 \omega+\mathrm{S}$ & 332.1 \\
55706.937 & $4 \mathrm{~d}^{4}\left({ }^{5} \mathrm{D}\right) 5 \mathrm{p} \mathrm{z}^{4} \mathrm{D}_{7 / 2}^{\circ}$ & 236.64 & $3 \omega+\mathrm{S}$ & 329.2 \\
57892.289 & $4 \mathrm{~d}^{4}\left({ }^{3} \mathrm{H}\right) 5 \mathrm{p} \mathrm{z}^{4} \mathrm{H}_{7 / 2}^{\circ}$ & 234.16 & $3 \omega+\mathrm{S}$ & 317.5 \\
58196.928 & $4 \mathrm{~d}^{4}\left({ }^{3} \mathrm{H}\right) 5 \mathrm{p} \mathrm{z}^{4} \mathrm{H}_{9 / 2}^{\circ}$ & 233.21 & $3 \omega+\mathrm{S}$ & 315.3 \\
58761.258 & $4 \mathrm{~d}^{4}\left({ }^{3} \mathrm{H}\right) 5 \mathrm{p} \mathrm{z}^{4} \mathrm{H}_{11 / 2}^{\circ}$ & 230.70 & $3 \omega+\mathrm{S}$ & 312.2 \\
59492.157 & $4 \mathrm{~d}^{4}\left({ }^{3} \mathrm{H}\right) 5 \mathrm{p} \mathrm{z}^{4} \mathrm{H}_{13 / 2}^{\circ}$ & 226.97 & $3 \omega+\mathrm{S}$ & 308.8 \\
59841.060 & $4 \mathrm{~d}^{4}\left({ }^{3} \mathrm{~F}\right) 5 \mathrm{p} \mathrm{z}^{2} \mathrm{D}_{3 / 2}^{\circ}$ & 223.94 & $3 \omega$ & 424.5 \\
60992.764 & $4 \mathrm{~d}^{4}\left({ }^{3} \mathrm{~F}\right) 5 \mathrm{p} \mathrm{z}^{2} \mathrm{D}_{5 / 2}^{\circ}$ & 218.93 & $3 \omega$ & 412.2 \\
\hline
\end{tabular}

${ }^{a}$ The level energies and designations are from Nilsson and Pickering (2003).

$3 \omega$ designates the third harmonics of the dye laser.

$\mathrm{S}$ and $2 \mathrm{~S}$ represent the first- and second-order Stokes components.

\section{Classified lines and term analysis in Mo II}

The ground level of Mo II is $4 d^{5} a^{6} S_{5 / 2}$. The level structure of this ion was first studied by Meggers and Kiess (1926) who established 27 levels. Later on, Schauls and Sawyer (1940) and Rau (1949) extended the analysis. An extensive experimental work on this ion was performed by Kiess (1958) who measured wavelengths of 3800 lines with uncertainties of $\pm 0.01 \AA$. The experimental values of the 238 excited levels reported in the NIST compilation (Sugar and Musgrove 1988) for the $4 \mathrm{~d}^{5}$, $4 d^{4} 5 s$ and $4 d^{4} 5 p$ configurations were taken from this analysis.

More recently, an extensive list of classified Mo II transitions between 152.9 and $721.7 \mathrm{~nm}$ has been reported by Nilsson (2002). These data were recorded with the Lund UV Fourier transform spectrometer (Sweden) and the Imperial College VUV Fourier transform spectrometer (UK). As a consequence, the term system of Mo II was extended by Nilsson and Pickering (2003) and energy values were reported for 330 levels (153 even and 177 odd levels) belonging to the $4 d^{5}, 4 d^{4} 5 s, 4 d^{3} 5 s^{2}, 4 d^{4} 6 s, 4 d^{4} 5 d, 4 d^{4} 5 p$ and $4 d^{3} 5 s 5 p$ configurations.

The energy levels, their designations as well as the wavelengths reported in the present paper are taken from Nilsson (2002) and Nilsson and Pickering (2003).

\section{Lifetime measurements}

In the present work, the lifetimes of 16 odd levels in the doublet, quartet and sextet systems of Mo II have been measured using the time-resolved laser-induced fluorescence (TR-LIF) technique applied to a laser-produced plasma.

The experimental setup used is similar to that described previously (Bergström et al 1988, Biémont et al 2001, Zhang et al 2001, Xu et al 2004) and only a brief description is presented here.
Free Mo II ions were obtained from a laser-produced plasma. Laser pulses characterized by a $532 \mathrm{~nm}$ wavelength, a $10 \mathrm{~Hz}$ repetition rate and a $10 \mathrm{~ns}$ duration were obtained from a Nd:YAG laser (Continuum Surelite). The pulses were focused vertically on the surface of a rotating pure molybdenum target in a vacuum chamber creating a plasma containing molybdenum atoms and ions in different ionization stages.

To populate the levels of interest, UV laser pulses were obtained in the following way. The $8 \mathrm{~ns}$ long output of an injection-seeded Nd:YAG laser (Continuum NY-82) with a repetition rate of $10 \mathrm{~Hz}$ and a single pulse energy of $400 \mathrm{~mJ}$ was reduced to 1-2 ns in a stimulated Brillouin scattering compressor. These pulses pumped a dye laser (Continuum Nd-60) with a DCM dye. The dye laser output was frequency doubled or tripled in a nonlinear optical system consisting of a KDP crystal, a retarding plate and a BBO crystal. According to the excitation schemes given in table 1, Stokes stimulated Raman scattering components were produced by focusing the harmonic radiation into a hydrogen conversion cell with a gas pressure of about 10 bar. The excitation laser beam was selected using a quartz Pellin-Broca prism and sent horizontally through the vacuum chamber where it interacted with the created molybdenum ions about $5 \mathrm{~mm}$ above the target.

The measured levels belong to the $\mathrm{z}^{4} \mathrm{P}^{\circ}, \mathrm{z}^{6} \mathrm{D}^{\circ}, \mathrm{z}{ }^{4} \mathrm{~F}^{\circ}$, $\mathrm{z}^{4} \mathrm{D}^{\circ}, \mathrm{z}^{4} \mathrm{H}^{\circ}$ and $\mathrm{z}^{2} \mathrm{D}^{\circ}$ odd terms. Of these levels, only $\mathrm{z}^{4} \mathrm{P}_{3 / 2}$ (at $48022 \mathrm{~cm}^{-1}$ ) was excited directly from the ground level. In all other cases we started from either the a ${ }^{6} \mathrm{D}$ or the a ${ }^{4} \mathrm{G}$ terms.

The fluorescence from the excited levels was imaged on the entrance slit of a $f=1 / 8$ monochromator and detected by a Hamamatsu R3809U photomultiplier with a risetime of $0.15 \mathrm{~ns}$. The time-resolved signal was averaged over 1000 laser pulses. The experimental lifetimes were extracted by a weighted least-squares fit of a single exponential decay, 
Table 2. Experimental and theoretical lifetimes (in ns) in Mo II.

\begin{tabular}{|c|c|c|c|c|c|}
\hline \multirow[b]{2}{*}{$\operatorname{Level}^{\mathrm{a}}\left(\mathrm{cm}^{-1}\right)$} & \multirow[b]{2}{*}{ Designation $^{\mathrm{a}}$} & \multicolumn{2}{|c|}{ Experiment } & \multicolumn{2}{|c|}{ Theory } \\
\hline & & Previous & This work & Previous & This work \\
\hline 45853.395 & $4 d^{4}\left({ }^{5} D\right) 5 p z^{6} F_{1 / 2}^{\circ}$ & $4.9(3)^{\mathrm{b}}$ & & $4.9^{\mathrm{d}}$ & 4.8 \\
\hline 46148.283 & $4 d^{4}\left({ }^{5} \mathrm{D}\right) 5 \mathrm{p} \mathrm{z}{ }^{6} \mathrm{~F}_{3 / 2}^{\circ}$ & $4.9(3)^{\mathrm{b}}$ & & $4.8^{\mathrm{d}}$ & 4.7 \\
\hline 46614.470 & $4 d^{4}\left({ }^{5} D\right) 5 p z^{6} F_{5 / 2}^{\circ}$ & $4.9(3)^{\mathrm{b}}$ & & $4.7^{\mathrm{d}}$ & 4.6 \\
\hline 47232.275 & $4 d^{4}\left({ }^{5} \mathrm{D}\right) 5 \mathrm{p} \mathrm{z}{ }^{6} \mathrm{~F}_{7 / 2}^{\circ}$ & $5.0(3)^{\mathrm{b}}$ & & $4.6^{\mathrm{d}}$ & 4.5 \\
\hline 47999.856 & $4 d^{4}\left({ }^{5} \mathrm{D}\right) 5 \mathrm{p} \mathrm{z}{ }^{6} \mathrm{~F}_{9 / 2}^{\circ}$ & $4.4(3)^{\mathrm{b}}$ & & $4.4^{\mathrm{d}}$ & 4.4 \\
\hline 48960.043 & $4 d^{4}\left({ }^{5} \mathrm{D}\right) 5 \mathrm{p} \mathrm{z}{ }^{6} \mathrm{~F}_{11 / 2}^{\circ}$ & $4.3(3)^{\mathrm{b}}$ & & $4.2^{\mathrm{d}}$ & 4.2 \\
\hline 48022.815 & $4 d^{4}\left({ }^{5} \mathrm{D}\right) 5 \mathrm{p} \mathrm{z}{ }^{4} \mathrm{P}_{3 / 2}^{\circ}$ & $4.8(4)^{b}, 4.9(3)^{c}$ & $4.9(2)$ & $5.1^{\mathrm{d}}$ & 4.8 \\
\hline 50577.720 & $4 d^{4}\left({ }^{5} D\right) 5 p z^{4} P_{5 / 2}^{\circ}$ & $4.7(4)^{b}, 4.5(3)^{c}$ & & $4.6^{\mathrm{d}}$ & 4.6 \\
\hline 49041.073 & 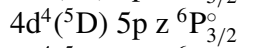 & $3.0(4)^{\mathrm{b}}, 2.2(2)^{\mathrm{c}}$ & & $2.1^{\mathrm{d}}$ & 2.1 \\
\hline 48860.829 & $4 d^{4}\left({ }^{5} \mathrm{D}\right) 5 \mathrm{p} \mathrm{z}{ }^{6} \mathrm{P}_{5 / 2}^{\circ}$ & $3.3(4)^{\mathrm{b}}, 2.8(2)^{\mathrm{c}}$ & & $3.0^{\mathrm{d}}$ & 2.8 \\
\hline 49481.300 & $4 d^{4}\left({ }^{5} \mathrm{D}\right) 5 \mathrm{p} \mathrm{z}{ }^{6} \mathrm{P}_{7 / 2}^{\circ}$ & $2.9(4)^{b}, 2.1(2)^{c}$ & & $2.1^{\mathrm{d}}$ & 2.1 \\
\hline 49949.756 & $4 d^{4}\left({ }^{5} \mathrm{D}\right) 5 \mathrm{p} \mathrm{z}{ }^{6} \mathrm{D}_{1 / 2}^{\circ}$ & $4.3(4)^{\mathrm{b}}, 4.3(3)^{\mathrm{b}}$ & & $4.5^{\mathrm{d}}$ & 4.3 \\
\hline 50192.337 & $4 d^{4}\left({ }^{5} D\right) 5 p z^{6} D_{3 / 2}^{\circ}$ & $4.3(3)^{\mathrm{c}}$ & & $4.5^{\mathrm{d}}$ & 4.4 \\
\hline 49609.086 & $4 d^{4}\left({ }^{5} \mathrm{D}\right) 5 \mathrm{p} \mathrm{z}{ }^{6} \mathrm{D}_{5 / 2}^{\circ}$ & $3.5(4)^{\mathrm{b}}, 2.9(2)^{\mathrm{c}}$ & & $2.9^{\mathrm{d}}$ & 3.1 \\
\hline 50302.913 & $4 d^{4}\left({ }^{5} \mathrm{D}\right) 5 \mathrm{p} \mathrm{z}{ }^{6} \mathrm{D}_{7 / 2}^{\circ}$ & $4.3(3)^{\mathrm{b}}, 4.0(3)^{\mathrm{c}}$ & $4.0(3)$ & $4.4^{\mathrm{d}}$ & 4.2 \\
\hline 50705.942 & $4 d^{4}\left({ }^{5} \mathrm{D}\right) 5 \mathrm{p} \mathrm{z}{ }^{6} \mathrm{D}_{9 / 2}^{\circ}$ & $4.3(3)^{\mathrm{b}}, 4.4(3)^{\mathrm{c}}$ & & $4.7^{\mathrm{d}}$ & 4.5 \\
\hline 51373.170 & $4 d^{4}\left({ }^{5} D\right) 5 p z^{4} F_{3 / 2}^{\circ}$ & & $4.2(3)$ & $4.6^{\mathrm{d}}$ & 4.7 \\
\hline 51732.690 & $4 d^{4}\left({ }^{5} D\right) 5 p z^{4} F_{5 / 2}^{\circ}$ & & $4.2(3)$ & $4.5^{\mathrm{d}}$ & 4.6 \\
\hline 52217.587 & $4 d^{4}\left({ }^{5} D\right) 5 p z^{4} F_{7 / 2}^{\circ}$ & & $4.0(3)$ & $4.4^{\mathrm{d}}$ & 4.4 \\
\hline 52843.296 & $4 d^{4}\left({ }^{5} \mathrm{D}\right) 5 \mathrm{p} \mathrm{z}{ }^{4} \mathrm{~F}_{9 / 2}^{\circ}$ & & $4.0(3)$ & $4.3^{\mathrm{d}}$ & 4.3 \\
\hline 54238.949 & $4 d^{4}\left({ }^{5} D\right) 5 p z^{4} D_{1 / 2}^{\circ}$ & & $5.1(3)$ & $5.8^{\mathrm{d}}$ & 5.5 \\
\hline 54687.002 & $4 d^{4}\left({ }^{5} D\right) 5 p z^{4} D_{3 / 2}^{\circ}$ & & $5.0(3)$ & $5.5^{\mathrm{d}}$ & 5.2 \\
\hline 55216.203 & $4 d^{4}\left({ }^{5} \mathrm{D}\right) 5 \mathrm{p} \mathrm{z}{ }^{4} \mathrm{D}_{5 / 2}^{\circ}$ & & $4.6(3)$ & $5.1^{\mathrm{d}}$ & 4.9 \\
\hline 55706.937 & $4 d^{4}\left({ }^{5} \mathrm{D}\right) 5 \mathrm{p} \mathrm{z}{ }^{4} \mathrm{D}_{7 / 2}^{\circ}$ & & $4.5(3)$ & $4.8^{\mathrm{d}}$ & 4.7 \\
\hline 57892.289 & $4 \mathrm{~d}^{4}\left({ }^{3} \mathrm{H}\right) 5 \mathrm{p} \mathrm{z}{ }^{4} \mathrm{H}_{7 / 2}^{\circ}$ & & $4.9(3)$ & $5.2^{\mathrm{d}}$ & 5.2 \\
\hline 58196.928 & $4 d^{4}\left({ }^{3} H\right) 5 p z^{4} H_{9 / 2}^{\circ}$ & & $4.9(3)$ & $5.2^{\mathrm{d}}$ & 5.2 \\
\hline 58761.258 & $4 d^{4}\left({ }^{3} \mathrm{H}\right) 5 \mathrm{p} \mathrm{z}{ }^{4} \mathrm{H}_{11 / 2}^{\circ}$ & & $4.8(3)$ & $5.0^{\mathrm{d}}$ & 5.0 \\
\hline 59492.157 & $4 d^{4}\left({ }^{3} \mathrm{H}\right) 5 \mathrm{p} \mathrm{z}{ }^{4} \mathrm{H}_{13 / 2}^{\circ}$ & & $4.6(3)$ & $4.8^{\mathrm{d}}$ & 4.7 \\
\hline 59841.060 & $4 d^{4}\left({ }^{3} F\right) 5 p z^{2} D_{3 / 2}^{\circ}$ & & $7.6(1.0)$ & $9.1^{\mathrm{d}}$ & 8.5 \\
\hline 60992.764 & $4 d^{4}\left({ }^{3} F\right) 5 p z^{2} D_{5 / 2}^{\circ}$ & & $6.6(5)$ & $7.4^{\mathrm{d}}$ & 7.5 \\
\hline
\end{tabular}

${ }^{\mathrm{a}}$ The level energies and designations are from Nilsson and Pickering (2003).

${ }^{\mathrm{b}}$ Hannaford and Lowe (1983).

${ }^{\text {c }}$ Sikström et al (2001).

${ }^{\text {d }}$ Quinet (2002) (Calculation $F$ ).

convoluted with the shape of the laser pulse, to the fluorescence signal. In addition, a polynomial background representation could be added in the fit. About ten curves were recorded and averaged for each level investigated. The 16 lifetimes obtained are given in table 2, where the error bars reflect not only the statistical uncertainties but also a conservative estimate of possible remaining systematic errors.

\section{Transition probabilities}

For the calculations of branching fractions we adopted the theoretical HFR model (Cowan 1981) previously described by Quinet (2002) (model $F$ in that work); see the discussion in the original paper. The computational procedure included the following configurations: $4 d^{5}, 4 d^{4} 5 s, 4 d^{4} 6 s, 4 d^{4} 5 d, 4 d^{3} 5 s^{2}$, $4 d^{3} 5 p^{2}, 4 d^{3} 5 d^{2}$ and $4 d^{3} 5 s 5 d$ (even parity) and $4 d^{4} 5 p, 4 d^{4} 6 p$, $4 d^{4} 4 f, 4 d^{4} 5 f, 4 d^{3} 5 s 5 p$ and $4 d^{3} 5 p 5 d$ (odd parity).

A $\mathrm{Mo}^{3+}$ ionic core of the type $[\mathrm{Ar}] 3 \mathrm{~d}^{10} 4 \mathrm{~s}^{2} 4 \mathrm{p}^{6} 4 \mathrm{~d}^{3}$ was considered with a value for the dipole polarizability, $\alpha_{d}$, equal to $5.67 \mathrm{a}_{0}^{3}$ (Fraga et al 1976) and a cut-off radius, $r_{c}=$ $1.73 a_{0}$. In addition, the final wavefunctions were obtained by a parametric fit of the Slater parameters using the experimental energy levels.

In the previous work of Quinet (2002), the optimization process was performed using the experimental energies compiled by Sugar and Musgrove (1988) for the $4 d^{5}, 4 d^{4} 5 \mathrm{~s}$ and $4 d^{4} 5 p$ configurations. Moreover, for the odd-parity levels the fit was limited to the lowest experimental values $\left(E<62000 \mathrm{~cm}^{-1}\right.$ ) because some levels, above that limit, had no spectroscopic designations in the NIST compilation.

In the present work, a new fit was carried out using the designations and experimental energy levels published by Nilsson and Pickering (2003). This allowed us to refine the parametric adjustment of the $4 d^{5}, 4 d^{4} 5 s$ and $4 d^{4} 5 p$ configurations and to extend it to the $4 d^{4} 6 s, 4 d^{4} 5 d$ and $4 d^{3} 5 s 5 p$ configurations. More precisely, for the even parity, all the levels of Nilsson and Pickering (2003) were used to fit the average energies $\left(E_{\mathrm{av}}\right)$, the direct $\left(F^{k}\right)$ and exchange $\left(G^{k}\right)$ electrostatic integrals and the spin-orbit $\left(\zeta_{n l}\right)$ parameters belonging to the $4 d^{5}, 4 d^{4} 5 s, 4 d^{4} 6 s$ and $4 d^{4} 5 d$ configurations. The configuration interaction integral $\left(R^{k}\right)$ between $4 d^{5}$ and $4 d^{4} 5 \mathrm{~s}$, together with effective interaction parameters $\alpha$ and $\beta$ 
Table 3. Transition probabilities for Mo II lines depopulating the levels for which the lifetimes have been measured in the present work. Only the most intense transitions ( $\log g f>-1.50)$ are quoted. $a(b)$ is written for $a \cdot 10^{b}$.

\begin{tabular}{|c|c|c|c|c|c|c|c|}
\hline$E_{\text {Low }}{ }^{\mathrm{a}}\left(\mathrm{cm}^{-1}\right)$ & Designation $^{a}$ & $E_{\text {Upp. }}{ }^{a}\left(\mathrm{~cm}^{-1}\right)$ & Designation $^{\mathrm{a}}$ & $\lambda^{\mathrm{b}}(\mathrm{nm})$ & $\log g f^{\mathrm{c}}$ & $g A^{\mathrm{c}}\left(\mathrm{s}^{-1}\right)$ & Int. $^{b}$ \\
\hline 12034.420 & $\left({ }^{5} \mathrm{D}\right) 5 \mathrm{~s}{ }^{6} \mathrm{D}_{3 / 2}$ & $47208.734^{\mathrm{d}}$ & $\left({ }^{5} \mathrm{D}\right) 5 \mathrm{p}{ }^{4} \mathrm{P}_{1 / 2}^{\circ}$ & 284.2148 & -0.92 & $9.86(7)$ & 61 \\
\hline 15699.390 & $4 d^{5}{ }^{4} P_{3 / 2}$ & $47208.734^{\mathrm{d}}$ & ( $\left.{ }^{5} \mathrm{D}\right) 5 \mathrm{p}^{4} \mathrm{P}_{1 / 2}^{\mathrm{o}}$ & 317.2744 & -0.90 & $8.28(7)$ & 169 \\
\hline 15890.422 & $4 d^{5}{ }^{4} P_{1 / 2}$ & $47208.734^{\mathrm{d}}$ & $\left({ }^{5} \mathrm{D}\right) 5 \mathrm{p}{ }^{4} \mathrm{P}_{1 / 2}^{\mathrm{o}}$ & 319.2097 & -1.25 & $3.67(7)$ & 83 \\
\hline 16796.468 & $4 d^{54} D_{1 / 2}$ & $47208.734^{\mathrm{d}}$ & $\left({ }^{5} \mathrm{D}\right) 5 \mathrm{p}{ }^{4} \mathrm{P}_{1 / 2}^{\circ}$ & 328.7200 & -1.38 & $2.56(7)$ & 57 \\
\hline 24372.408 & $\left({ }^{5} D\right) 5 s{ }^{4} D_{1 / 2}$ & $47208.734^{\mathrm{d}}$ & ( $\left.{ }^{5} \mathrm{D}\right) 5 \mathrm{p}^{4} \mathrm{P}_{1 / 2}^{\mathrm{o}}$ & 437.7758 & -1.08 & $2.90(7)$ & 93 \\
\hline 24659.500 & $\left({ }^{5} D\right) 5 s{ }^{4} D_{3 / 2}$ & $47208.734^{\mathrm{d}}$ & $\left({ }^{5} \mathrm{D}\right) 5 \mathrm{p}{ }^{4} \mathrm{P}_{1 / 2}^{\circ}$ & 443.3496 & -1.14 & $2.47(7)$ & 85 \\
\hline 0.000 & $4 d^{56} S_{5 / 2}$ & 48022.815 & $\left({ }^{5} \mathrm{D}\right) 5 \mathrm{p}{ }^{4} \mathrm{P}_{3 / 2}^{\circ}$ & 208.1681 & -1.15 & $1.10(8)$ & 53 \\
\hline 12417.632 & $\left({ }^{5} \mathrm{D}\right) 5 \mathrm{~s}{ }^{6} \mathrm{D}_{5 / 2}$ & 48022.815 & $\left({ }^{5} \mathrm{D}\right) 5 \mathrm{p}{ }^{4} \mathrm{P}_{3 / 2}^{\circ}$ & 280.7753 & -0.43 & $3.13(8)$ & 222 \\
\hline 15691.451 & $4 d^{54} P_{5 / 2}$ & 48022.815 & $\left({ }^{5} \mathrm{D}\right) 5 \mathrm{p}{ }^{4} \mathrm{P}_{3 / 2}^{\circ}$ & 309.2074 & -0.75 & $1.25(8)$ & 212 \\
\hline 16796.468 & $4 d^{5}{ }^{4} D_{1 / 2}$ & 48022.815 & $\left({ }^{5} \mathrm{D}\right) 5 \mathrm{p}{ }^{4} \mathrm{P}_{3 / 2}^{\circ}$ & 320.1499 & -1.34 & $2.97(7)$ & 57 \\
\hline 17174.332 & $4 d^{5}{ }^{4} D_{3 / 2}$ & 48022.815 & $\left({ }^{5} \mathrm{D}\right) 5 \mathrm{p}^{4} \mathrm{P}_{3 / 2}^{\circ}$ & 324.0715 & -0.95 & 7.13(7) & 152 \\
\hline 17344.363 & $4 d^{5}{ }^{4} D_{5 / 2}$ & 48022.815 & $\left({ }^{5} \mathrm{D}\right) 5 \mathrm{p}{ }^{4} \mathrm{P}_{3 / 2}^{\circ}$ & 325.8677 & -1.40 & $2.49(7)$ & 55 \\
\hline 24659.500 & $\left({ }^{5} \mathrm{D}\right) 5 \mathrm{~s}{ }^{4} \mathrm{D}_{3 / 2}$ & 48022.815 & $\left({ }^{5} \mathrm{D}\right) 5 \mathrm{p}^{4} \mathrm{P}_{3 / 2}^{\mathrm{o}}$ & 427.9011 & -1.00 & $3.67(7)$ & 117 \\
\hline 25112.565 & $\left({ }^{5} \mathrm{D}\right) 5 \mathrm{~s}{ }^{4} \mathrm{D}_{5 / 2}$ & 48022.815 & $\left({ }^{5} \mathrm{D}\right) 5 \mathrm{p}^{4} \mathrm{P}_{3 / 2}^{\circ}$ & 436.3632 & -0.96 & $3.87(7)$ & 119 \\
\hline 12417.632 & $\left({ }^{5} \mathrm{D}\right) 5 \mathrm{~s}{ }^{6} \mathrm{D}_{5 / 2}$ & 50302.913 & ( $\left.{ }^{5} \mathrm{D}\right) 5 \mathrm{p}^{6} \mathrm{D}_{7 / 2}^{\circ}$ & 263.8761 & -0.07 & $8.25(8)$ & 948 \\
\hline 12900.680 & $\left({ }^{5} \mathrm{D}\right) 5 \mathrm{~s}{ }^{6} \mathrm{D}_{7 / 2}$ & 50302.913 & $\left({ }^{5} \mathrm{D}\right) 5 \mathrm{p}{ }^{6} \mathrm{D}_{7 / 2}^{\circ}$ & 267.2843 & -0.06 & $8.06(8)$ & 878 \\
\hline 13461.113 & $\left({ }^{5} \mathrm{D}\right) 5 \mathrm{~s}{ }^{6} \mathrm{D}_{9 / 2}$ & 50302.913 & $\left({ }^{5} \mathrm{D}\right) 5 \mathrm{p}{ }^{6} \mathrm{D}_{7 / 2}^{\circ}$ & 271.3504 & -0.82 & $1.38(8)$ & 119 \\
\hline 25112.565 & $\left({ }^{5} \mathrm{D}\right) 5 \mathrm{~s}{ }^{4} \mathrm{D}_{5 / 2}$ & 50302.913 & $\left({ }^{5} \mathrm{D}\right) 5 \mathrm{p}{ }^{6} \mathrm{D}_{7 / 2}^{\circ}$ & 396.8651 & -1.32 & $2.03(7)$ & 59 \\
\hline 15199.383 & $4 d^{54} G_{5 / 2}$ & 51373.170 & $\left({ }^{5} \mathrm{D}\right) 5 \mathrm{p}^{4} \mathrm{~F}_{3 / 2}^{\circ}$ & 276.3616 & -0.61 & $2.18(8)$ & 182 \\
\hline 15699.390 & $4 d^{5}{ }^{4} \mathrm{P}_{3 / 2}$ & 51373.170 & ( $\left.{ }^{5} \mathrm{D}\right) 5 \mathrm{p}^{4} \mathrm{~F}_{3 / 2}^{\mathrm{o}}$ & 280.2353 & -1.33 & $4.05(7)$ & 27 \\
\hline 15890.422 & $4 d^{5}{ }^{4} P_{1 / 2}$ & 51373.170 & $\left({ }^{5} \mathrm{D}\right) 5 \mathrm{p}^{4} \mathrm{~F}_{3 / 2}^{\circ}$ & 281.7441 & -1.11 & $6.50(7)$ & 39 \\
\hline 16796.468 & $4 d^{5}{ }^{4} D_{1 / 2}$ & 51373.170 & $\left({ }^{5} \mathrm{D}\right) 5 \mathrm{p}^{4} \mathrm{~F}_{3 / 2}^{\circ}$ & 289.1273 & -0.73 & $1.49(8)$ & 33 \\
\hline 17174.332 & $4 d^{54} D_{3 / 2}$ & 51373.170 & $\left({ }^{5} \mathrm{D}\right) 5 \mathrm{p}^{4} \mathrm{~F}_{3 / 2}^{\circ}$ & 292.3220 & -1.13 & $5.83(7)$ & 43 \\
\hline 24137.912 & $4 d^{5}{ }^{4} F_{3 / 2}$ & 51373.170 & $\left({ }^{5} \mathrm{D}\right) 5 \mathrm{p}^{4} \mathrm{~F}_{3 / 2}^{\circ}$ & 367.0666 & -1.11 & $3.90(7)$ & 49 \\
\hline 24372.408 & $\left({ }^{5} \mathrm{D}\right) 5 \mathrm{~s}{ }^{4} \mathrm{D}_{1 / 2}$ & 51373.170 & $\left({ }^{5} \mathrm{D}\right) 5 \mathrm{p}^{4} \mathrm{~F}_{3 / 2}^{\circ}$ & 370.2546 & -0.44 & $1.79(8)$ & 262 \\
\hline 24659.500 & ( $\left.{ }^{5} \mathrm{D}\right) 5 \mathrm{~s}{ }^{4} \mathrm{D}_{3 / 2}$ & 51373.170 & $\left({ }^{5} \mathrm{D}\right) 5 \mathrm{p}^{4} \mathrm{~F}_{3 / 2}^{\circ}$ & 374.2338 & -0.89 & $6.23(7)$ & 93 \\
\hline 15330.789 & $4 d^{5}{ }^{4} G_{7 / 2}$ & 51732.690 & $\left({ }^{5} \mathrm{D}\right) 5 \mathrm{p}^{4} \mathrm{~F}_{5 / 2}^{\circ}$ & 274.6297 & -0.48 & $2.94(8)$ & 271 \\
\hline 15691.451 & $4 d^{5}{ }^{4} P_{5 / 2}$ & 51732.690 & $\left({ }^{5} \mathrm{D}\right) 5 \mathrm{p}^{4} \mathrm{~F}_{5 / 2}^{\circ}$ & 277.3780 & -1.19 & $5.58(7)$ & 43 \\
\hline 15699.390 & $4 d^{5}{ }^{4} P_{3 / 2}$ & 51732.690 & $\left({ }^{5} \mathrm{D}\right) 5 \mathrm{p}^{4} \mathrm{~F}_{5 / 2}^{\circ}$ & 277.4392 & -0.78 & $1.43(8)$ & 122 \\
\hline 17174.332 & $4 d^{5}{ }^{4} D_{3 / 2}$ & 51732.690 & $\left({ }^{5} \mathrm{D}\right) 5 \mathrm{p}^{4} \mathrm{~F}_{5 / 2}^{\mathrm{o}}$ & 289.2808 & -0.66 & $1.75(8)$ & 48 \\
\hline 17344.363 & $4 d^{54} D_{5 / 2}$ & 51732.690 & $\left({ }^{5} \mathrm{D}\right) 5 \mathrm{p}^{4} \mathrm{~F}_{5 / 2}^{\circ}$ & 290.7112 & -0.96 & $8.82(7)$ & 74 \\
\hline 23934.630 & $4 d^{5}{ }^{4} F_{5 / 2}$ & 51732.690 & $\left({ }^{5} \mathrm{D}\right) 5 \mathrm{p}^{4} \mathrm{~F}_{5 / 2}^{\circ}$ & 359.6347 & -0.97 & $5.56(7)$ & 71 \\
\hline 24659.500 & ( $\left.{ }^{5} \mathrm{D}\right) 5 \mathrm{~s}{ }^{4} \mathrm{D}_{3 / 2}$ & 51732.690 & $\left({ }^{5} \mathrm{D}\right) 5 \mathrm{p}^{4} \mathrm{~F}_{5 / 2}^{\circ}$ & 369.2640 & -0.24 & $2.85(8)$ & 446 \\
\hline 25112.565 & ( $\left.{ }^{5} \mathrm{D}\right) 5 \mathrm{~s}{ }^{4} \mathrm{D}_{5 / 2}$ & 51732.690 & $\left({ }^{5} \mathrm{D}\right) 5 \mathrm{p}^{4} \mathrm{~F}_{5 / 2}^{\circ}$ & 375.5489 & -0.91 & $5.88(7)$ & 94 \\
\hline 12900.688 & $4 d^{5}{ }^{6} D_{7 / 2}$ & 52217.587 & ( $\left.{ }^{5} \mathrm{D}\right) 5 \mathrm{p}^{4} \mathrm{~F}_{7 / 2}^{\circ}$ & 254.2672 & -0.84 & $1.50(8)$ & 129 \\
\hline 15427.895 & $4 d^{5}{ }^{4} G_{9 / 2}$ & 52217.587 & $\left({ }^{5} \mathrm{D}\right) 5 \mathrm{p}^{4} \mathrm{~F}_{7 / 2}^{\circ}$ & 271.7348 & -0.37 & $3.87(8)$ & 376 \\
\hline 15691.451 & $4 d^{5}{ }^{4} P_{5 / 2}$ & 52217.587 & $\left({ }^{5} \mathrm{D}\right) 5 \mathrm{p}^{4} \mathrm{~F}_{7 / 2}^{\circ}$ & 273.6956 & -0.71 & $1.74(8)$ & 169 \\
\hline 16947.078 & $4 d^{5}{ }^{4} D_{7 / 2}$ & 52217.587 & $\left({ }^{5} \mathrm{D}\right) 5 \mathrm{p}^{4} \mathrm{~F}_{7 / 2}^{\circ}$ & 283.4396 & -0.90 & $1.05(8)$ & 47 \\
\hline 17344.363 & $4 d^{5}{ }^{4} D_{5 / 2}$ & 52217.587 & $\left({ }^{5} \mathrm{D}\right) 5 \mathrm{p}{ }^{4} \mathrm{~F}_{7 / 2}^{\circ}$ & 286.6688 & -0.49 & $2.66(8)$ & 84 \\
\hline 23853.578 & $4 d^{5}{ }^{4} F_{7 / 2}$ & 52217.587 & $\left({ }^{5} \mathrm{D}\right) 5 \mathrm{p}^{4} \mathrm{~F}_{7 / 2}^{\circ}$ & 352.4587 & -0.73 & $9.95(7)$ & 169 \\
\hline 23934.630 & $4 d^{5}{ }^{4} F_{5 / 2}$ & 52217.587 & $\left({ }^{5} \mathrm{D}\right) 5 \mathrm{p}^{4} \mathrm{~F}_{7 / 2}^{\circ}$ & 353.4686 & -1.30 & $2.70(7)$ & 74 \\
\hline 25112.565 & $\left({ }^{5} \mathrm{D}\right) 5 \mathrm{~s}{ }^{4} \mathrm{D}_{5 / 2}$ & 52217.587 & $\left({ }^{5} \mathrm{D}\right) 5 \mathrm{p}^{4} \mathrm{~F}_{7 / 2}^{\circ}$ & 368.8303 & -0.18 & $3.30(8)$ & 479 \\
\hline 25341.898 & ( $\left.{ }^{5} \mathrm{D}\right) 5 \mathrm{~s}{ }^{4} \mathrm{D}_{7 / 2}$ & 52217.587 & $\left({ }^{5} \mathrm{D}\right) 5 \mathrm{p}^{4} \mathrm{~F}_{7 / 2}^{\circ}$ & 371.9777 & -1.13 & $3.60(7)$ & 57 \\
\hline 13461.113 & $4 d^{56} D_{9 / 2}$ & 52843.296 & $\left({ }^{5} \mathrm{D}\right) 5 \mathrm{p}^{4} \mathrm{~F}_{9 / 2}^{\circ}$ & 253.8457 & -0.37 & $4.42(8)$ & 300 \\
\hline 15447.043 & $4 d^{5}{ }^{4} G_{11 / 2}$ & 52843.296 & $\left({ }^{5} \mathrm{D}\right) 5 \mathrm{p}^{4} \mathrm{~F}_{9 / 2}^{\circ}$ & 267.3270 & -0.29 & $4.84(8)$ & 433 \\
\hline 16947.078 & $4 d^{54} D_{7 / 2}$ & 52843.296 & $\left({ }^{5} \mathrm{D}\right) 5 \mathrm{p}^{4} \mathrm{~F}_{9 / 2}^{\circ}$ & 278.4987 & -0.19 & $5.59(8)$ & 327 \\
\hline 23833.154 & $4 d^{5}{ }^{4} F_{9 / 2}$ & 52843.296 & $\left({ }^{5} \mathrm{D}\right) 5 \mathrm{p}^{4} \mathrm{~F}_{9 / 2}^{\circ}$ & 344.6083 & -0.54 & $1.63(8)$ & 176 \\
\hline 25341.898 & $\left({ }^{5} \mathrm{D}\right) 5 \mathrm{~s}{ }^{4} \mathrm{D}_{7 / 2}$ & 52843.296 & $\left({ }^{5} \mathrm{D}\right) 5 \mathrm{p}^{4} \mathrm{~F}_{9 / 2}^{\circ}$ & 363.5143 & 0.04 & $5.56(8)$ & 579 \\
\hline 22864.466 & $4 d^{5}{ }^{2} D_{3 / 2}$ & 54238.949 & $\left({ }^{5} \mathrm{D}\right) 5 \mathrm{p}{ }^{4} \mathrm{D}_{1 / 2}^{\circ}$ & 318.6382 & -1.22 & $3.95(7)$ & 51 \\
\hline 24137.912 & $4 d^{5}{ }^{4} F_{3 / 2}$ & 54238.949 & ( $\left.{ }^{5} \mathrm{D}\right) 5 \mathrm{p}^{4} \mathrm{D}_{1 / 2}^{\circ}$ & 332.1189 & -1.20 & $3.79(7)$ & 46 \\
\hline 24372.408 & ( $\left.{ }^{5} \mathrm{D}\right) 5 \mathrm{~s}{ }^{4} \mathrm{D}_{1 / 2}$ & 54238.949 & $\left({ }^{5} \mathrm{D}\right) 5 \mathrm{p}^{4} \mathrm{D}_{1 / 2}^{\circ}$ & 334.7267 & -0.70 & $1.17(8)$ & 137 \\
\hline 24659.500 & ( $\left.{ }^{5} \mathrm{D}\right) 5 \mathrm{~s}{ }^{4} \mathrm{D}_{3 / 2}$ & 54238.949 & $\left({ }^{5} \mathrm{D}\right) 5 \mathrm{p}^{4} \mathrm{D}_{1 / 2}^{\circ}$ & 337.9755 & -0.68 & $1.21(8)$ & 151 \\
\hline 23934.630 & $4 d^{5}{ }^{4} F_{5 / 2}$ & 54687.002 & $\left({ }^{5} \mathrm{D}\right) 5 \mathrm{p}{ }^{4} \mathrm{D}_{3 / 2}^{\circ}$ & 325.0738 & -0.79 & $1.01(8)$ & 107 \\
\hline 24372.408 & $\left({ }^{5} \mathrm{D}\right) 5 \mathrm{~s}{ }^{4} \mathrm{D}_{1 / 2}$ & 54687.002 & $\left({ }^{5} \mathrm{D}\right) 5 \mathrm{p}^{4} \mathrm{D}_{3 / 2}^{\circ}$ & 329.7683 & -0.81 & $9.56(7)$ & 97 \\
\hline 24659.500 & ( $\left.{ }^{5} \mathrm{D}\right) 5 \mathrm{~s}{ }^{4} \mathrm{D}_{3 / 2}$ & 54687.002 & ( $\left.{ }^{5} \mathrm{D}\right) 5 \mathrm{p}^{4} \mathrm{D}_{3 / 2}^{\circ}$ & 332.9212 & -0.46 & $2.09(8)$ & 233 \\
\hline 25112.565 & ( $\left.{ }^{5} \mathrm{D}\right) 5 \mathrm{~s}{ }^{4} \mathrm{D}_{5 / 2}$ & 54687.002 & $\left({ }^{5} \mathrm{D}\right) 5 \mathrm{p}^{4} \mathrm{D}_{3 / 2}^{\circ}$ & 338.0213 & -0.57 & $1.55(8)$ & 165 \\
\hline 26603.864 & $\left({ }^{3} \mathrm{P}\right) 5 \mathrm{~s}{ }^{4} \mathrm{P}_{1 / 2}$ & 54687.002 & $\left({ }^{5} \mathrm{D}\right) 5 \mathrm{p}{ }^{4} \mathrm{D}_{3 / 2}^{\circ}$ & 355.9713 & -1.38 & $2.20(7)$ & 22 \\
\hline
\end{tabular}


Table 3. (Continued.)

\begin{tabular}{|c|c|c|c|c|c|c|c|}
\hline$E_{\text {Low }}{ }^{\mathrm{a}}\left(\mathrm{cm}^{-1}\right)$ & Designation $^{\mathrm{a}}$ & $E_{\text {Upp. }}{ }^{\mathrm{a}}\left(\mathrm{cm}^{-1}\right)$ & Designation $^{a}$ & $\lambda^{b}(\mathrm{~nm})$ & $\log g f^{\mathrm{c}}$ & $g A^{\mathrm{c}}\left(\mathrm{s}^{-1}\right)$ & Int. $^{b}$ \\
\hline 23853.578 & $4 d^{5}{ }^{4} F_{7 / 2}$ & 55216.203 & $\left({ }^{5} \mathrm{D}\right) 5 \mathrm{p}{ }^{4} \mathrm{D}_{5 / 2}^{\circ}$ & 318.7587 & -0.62 & $1.56(8)$ & 136 \\
\hline 23934.630 & $4 d^{5}{ }^{4} F_{5 / 2}$ & 55216.203 & $\left({ }^{5} \mathrm{D}\right) 5 \mathrm{p}^{4} \mathrm{D}_{5 / 2}^{\circ}$ & 319.5847 & -1.22 & $3.92(7)$ & 30 \\
\hline 24659.500 & $\left({ }^{5} \mathrm{D}\right) 5 \mathrm{~s}^{4} \mathrm{D}_{3 / 2}$ & 55216.203 & $\left({ }^{5} \mathrm{D}\right) 5 \mathrm{p}^{4} \mathrm{D}_{5 / 2}^{\circ}$ & 327.1662 & -0.64 & $1.41(8)$ & 135 \\
\hline 24836.319 & $4 d^{5}{ }^{2} F_{5 / 2}$ & 55216.203 & ( $\left.{ }^{5} \mathrm{D}\right) 5 \mathrm{p}^{4} \mathrm{D}_{5 / 2}^{\circ}$ & 329.0704 & -0.74 & $1.12(8)$ & 127 \\
\hline 25112.565 & $\left({ }^{5} \mathrm{D}\right) 5 \mathrm{~s}{ }^{4} \mathrm{D}_{5 / 2}$ & 55216.203 & $\left({ }^{5} \mathrm{D}\right) 5 \mathrm{p}^{4} \mathrm{D}_{5 / 2}^{\circ}$ & 332.0902 & -0.23 & $3.58(8)$ & 329 \\
\hline 25341.898 & ( $\left.{ }^{5} \mathrm{D}\right) 5 \mathrm{~s}{ }^{4} \mathrm{D}_{7 / 2}$ & 55216.203 & ( $\left.{ }^{5} \mathrm{D}\right) 5 \mathrm{p}^{4} \mathrm{D}_{5 / 2}^{\circ}$ & 334.6396 & -0.43 & $2.19(8)$ & 206 \\
\hline 27627.858 & $\left({ }^{3} \mathrm{P}\right) 5 \mathrm{~s}{ }^{4} \mathrm{P}_{3 / 2}$ & 55216.203 & $\left({ }^{5} \mathrm{D}\right) 5 \mathrm{p}^{4} \mathrm{D}_{5 / 2}^{\circ}$ & 362.3685 & -1.19 & $3.27(7)$ & 26 \\
\hline 15691.451 & $4 d^{5}{ }^{4} P_{5 / 2}$ & 55706.937 & $\left({ }^{5} \mathrm{D}\right) 5 \mathrm{p}{ }^{4} \mathrm{D}_{7 / 2}^{\circ}$ & 249.8279 & -1.13 & 7.79(7) & 58 \\
\hline 23833.154 & $4 d^{5}{ }^{4} F_{9 / 2}$ & 55706.937 & $\left({ }^{5} \mathrm{D}\right) 5 \mathrm{p}^{4} \mathrm{D}_{7 / 2}^{\circ}$ & 313.6466 & -0.46 & $2.35(8)$ & 159 \\
\hline 23853.578 & $4 d^{5}{ }^{4} F_{7 / 2}$ & 55706.937 & ( $\left.{ }^{5} \mathrm{D}\right) 5 \mathrm{p}^{4} \mathrm{D}_{7 / 2}^{\circ}$ & 313.8477 & -1.10 & $5.37(7)$ & 31 \\
\hline 25112.565 & $\left({ }^{5} \mathrm{D}\right) 5 \mathrm{~s}{ }^{4} \mathrm{D}_{5 / 2}$ & 55706.937 & $\left({ }^{5} \mathrm{D}\right) 5 \mathrm{p}^{4} \mathrm{D}_{7 / 2}^{\circ}$ & 326.7633 & -0.81 & $9.73(7)$ & 77 \\
\hline 25341.898 & $\left({ }^{5} \mathrm{D}\right) 5 \mathrm{~s}{ }^{4} \mathrm{D}_{7 / 2}$ & 55706.937 & $\left({ }^{5} \mathrm{D}\right) 5 \mathrm{p}{ }^{4} \mathrm{D}_{7 / 2}^{\circ}$ & 329.2313 & 0.18 & $9.28(8)$ & 765 \\
\hline 29022.466 & $\left({ }^{3} \mathrm{P}\right) 5 \mathrm{~s}{ }^{4} \mathrm{P}_{5 / 2}$ & 55706.937 & $\left({ }^{5} \mathrm{D}\right) 5 \mathrm{p}^{4} \mathrm{D}_{7 / 2}^{\circ}$ & 374.6433 & -1.13 & $3.53(7)$ & 25 \\
\hline 29034.426 & $\left({ }^{3} \mathrm{~F}\right) 5 \mathrm{~s}{ }^{4} \mathrm{~F}_{9 / 2}$ & 55706.937 & ( $\left.{ }^{5} \mathrm{D}\right) 5 \mathrm{p}^{4} \mathrm{D}_{7 / 2}^{\circ}$ & 374.8113 & -1.07 & $4.05(7)$ & 38 \\
\hline 15199.383 & $4 d^{5}{ }^{4} G_{5 / 2}$ & 57892.289 & $\left({ }^{3} \mathrm{H}\right) 5 \mathrm{p}^{4} \mathrm{H}_{7 / 2}^{\circ}$ & 234.1592 & -0.58 & $3.19(8)$ & 84 \\
\hline 26041.359 & $\left({ }^{3} \mathrm{H}\right) 5 \mathrm{~s}{ }^{4} \mathrm{H}_{7 / 2}$ & 57892.289 & $\left({ }^{3} \mathrm{H}\right) 5 \mathrm{p}^{4} \mathrm{H}_{7 / 2}^{\circ}$ & 313.8717 & -0.14 & $4.90(8)$ & 82 \\
\hline 26405.839 & $4 d^{52} G_{7 / 2}$ & 57892.289 & $\left({ }^{3} \mathrm{H}\right) 5 \mathrm{p}^{4} \mathrm{H}_{7 / 2}^{\circ}$ & 317.5051 & -0.32 & $3.20(8)$ & 89 \\
\hline 26488.470 & $\left({ }^{3} \mathrm{H}\right) 5 \mathrm{~s}{ }^{4} \mathrm{H}_{9 / 2}$ & 57892.289 & $\left({ }^{3} \mathrm{H}\right) 5 \mathrm{p}^{4} \mathrm{H}_{7 / 2}^{\circ}$ & 318.3405 & -1.04 & $5.98(7)$ & 11 \\
\hline 28877.238 & $\left({ }^{3} \mathrm{~F}\right) 5 \mathrm{~s}{ }^{4} \mathrm{~F}_{5 / 2}$ & 57892.289 & $\left({ }^{3} \mathrm{H}\right) 5 \mathrm{p}^{4} \mathrm{H}_{7 / 2}^{\circ}$ & 344.5500 & -0.63 & $1.33(8)$ & 38 \\
\hline 29699.468 & $\left({ }^{3} \mathrm{G}\right) 5 \mathrm{~s}^{4} \mathrm{G}_{5 / 2}$ & 57892.289 & $\left({ }^{3} \mathrm{H}\right) 5 \mathrm{p}^{4} \mathrm{H}_{7 / 2}^{\circ}$ & 354.5990 & -1.00 & $5.35(7)$ & 14 \\
\hline 15427.895 & $4 d^{5}{ }^{4} G_{9 / 2}$ & 58196.928 & $\left({ }^{3} \mathrm{H}\right) 5 \mathrm{p}^{4} \mathrm{H}_{9 / 2}^{\circ}$ & 233.7424 & -1.29 & $6.25(7)$ & 17 \\
\hline 15330.789 & $4 d^{5}{ }^{4} G_{7 / 2}$ & 58196.928 & $\left({ }^{3} \mathrm{H}\right) 5 \mathrm{p}^{4} \mathrm{H}_{9 / 2}^{\circ}$ & 233.2128 & -0.56 & $3.35(8)$ & 82 \\
\hline 26068.808 & $4 d^{5}{ }^{2} G_{9 / 2}$ & 58196.928 & $\left({ }^{3} \mathrm{H}\right) 5 \mathrm{p}^{4} \mathrm{H}_{9 / 2}^{\circ}$ & 311.1636 & -1.13 & $5.06(7)$ & 21 \\
\hline 26405.839 & $4 d^{52} G_{7 / 2}$ & 58196.928 & $\left({ }^{3} \mathrm{H}\right) 5 \mathrm{p}^{4} \mathrm{H}_{9 / 2}^{\circ}$ & 314.4625 & -1.29 & $3.46(7)$ & 10 \\
\hline 26488.470 & $\left({ }^{3} \mathrm{H}\right) 5 \mathrm{~s}{ }^{4} \mathrm{H}_{9 / 2}$ & 58196.928 & $\left({ }^{3} \mathrm{H}\right) 5 \mathrm{p}^{4} \mathrm{H}_{9 / 2}^{\circ}$ & 315.2820 & 0.18 & $1.03(9)$ & 197 \\
\hline 26739.737 & $\left({ }^{3} \mathrm{H}\right) 5 \mathrm{~s}{ }^{4} \mathrm{H}_{11 / 2}$ & 58196.928 & $\left({ }^{3} \mathrm{H}\right) 5 \mathrm{p}{ }^{4} \mathrm{H}_{9 / 2}^{\circ}$ & 317.8004 & -0.94 & 7.67(7) & 12 \\
\hline 30019.626 & $\left({ }^{3} G\right) 5 s{ }^{4} G_{7 / 2}$ & 58196.928 & $\left({ }^{3} \mathrm{H}\right) 5 \mathrm{p}^{4} \mathrm{H}_{9 / 2}^{\circ}$ & 354.7942 & -1.00 & $5.26(7)$ & 15 \\
\hline 15447.043 & $4 d^{5}{ }^{4} G_{11 / 2}$ & 58761.258 & $\left({ }^{3} \mathrm{H}\right) 5 \mathrm{p}^{4} \mathrm{H}_{11 / 2}^{\circ}$ & 230.8001 & -1.25 & $7.02(7)$ & 16 \\
\hline 15427.895 & $4 d^{5}{ }^{4} G_{9 / 2}$ & 58761.258 & $\left({ }^{3} \mathrm{H}\right) 5 \mathrm{p}^{4} \mathrm{H}_{11 / 2}^{\circ}$ & 230.6981 & -0.44 & $4.55(8)$ & 93 \\
\hline 26488.470 & $\left({ }^{3} \mathrm{H}\right) 5 \mathrm{~s}{ }^{4} \mathrm{H}_{9 / 2}$ & 58761.258 & $\left({ }^{3} \mathrm{H}\right) 5 \mathrm{p}{ }^{4} \mathrm{H}_{11 / 2}^{\circ}$ & 309.7687 & -0.81 & $1.07(8)$ & 24 \\
\hline 26739.737 & $\left({ }^{3} \mathrm{H}\right) 5 \mathrm{~s}{ }^{4} \mathrm{H}_{11 / 2}$ & 58761.258 & $\left({ }^{3} \mathrm{H}\right) 5 \mathrm{p}^{4} \mathrm{H}_{11 / 2}^{\circ}$ & 312.1995 & 0.32 & $1.43(9)$ & 216 \\
\hline 27114.158 & $\left({ }^{3} \mathrm{H}\right) 5 \mathrm{~s}{ }^{4} \mathrm{H}_{13 / 2}$ & 58761.258 & $\left({ }^{3} \mathrm{H}\right) 5 \mathrm{p}^{4} \mathrm{H}_{11 / 2}^{\circ}$ & 315.8932 & -1.03 & $6.17(7)$ & 8 \\
\hline 29034.426 & $\left({ }^{3} \mathrm{~F}\right) 5 \mathrm{~s}{ }^{4} \mathrm{~F}_{9 / 2}$ & 58761.258 & $\left({ }^{3} \mathrm{H}\right) 5 \mathrm{p}^{4} \mathrm{H}_{11 / 2}^{\circ}$ & 336.2998 & -0.70 & $1.19(8)$ & 23 \\
\hline 30213.841 & $\left({ }^{3} G\right) 5 s{ }^{4} G_{9 / 2}$ & 58761.258 & $\left({ }^{3} \mathrm{H}\right) 5 \mathrm{p}^{4} \mathrm{H}_{11 / 2}^{\circ}$ & 350.1942 & -0.72 & $1.04(8)$ & 25 \\
\hline 26739.737 & $\left({ }^{3} \mathrm{H}\right) 5 \mathrm{~s}{ }^{4} \mathrm{H}_{11 / 2}$ & 59492.157 & $\left({ }^{3} \mathrm{H}\right) 5 \mathrm{p}{ }^{4} \mathrm{H}_{13 / 2}^{\circ}$ & 305.2322 & -0.82 & $1.08(8)$ & 17 \\
\hline 27114.158 & $\left({ }^{3} \mathrm{H}\right) 5 \mathrm{~s}{ }^{4} \mathrm{H}_{13 / 2}$ & 59492.157 & $\left({ }^{3} \mathrm{H}\right) 5 \mathrm{p}^{4} \mathrm{H}_{13 / 2}^{\circ}$ & 308.7621 & 0.43 & $1.89(9)$ & 213 \\
\hline 30391.605 & $\left({ }^{3} G\right) 5 s{ }^{4} G_{11 / 2}$ & 59492.157 & $\left({ }^{3} \mathrm{H}\right) 5 \mathrm{p}^{4} \mathrm{H}_{13 / 2}^{\circ}$ & 343.5376 & -0.43 & $2.06(8)$ & 35 \\
\hline 33601.411 & $\left({ }^{3} \mathrm{H}\right) 5 \mathrm{~s}{ }^{2} \mathrm{H}_{11 / 2}$ & 59492.157 & $\left({ }^{3} \mathrm{H}\right) 5 \mathrm{p}^{4} \mathrm{H}_{13 / 2}^{\circ}$ & 386.1289 & -1.06 & $3.91(7)$ & 67 \\
\hline 22864.466 & $4 d^{52} D_{3 / 2}$ & 59841.060 & $\left({ }^{3} F\right) 5 p^{2} D_{3 / 2}^{\circ}$ & 270.3612 & -1.21 & $5.64(7)$ & 19 \\
\hline 26603.864 & $\left({ }^{3} \mathrm{P}\right) 5 \mathrm{~s}{ }^{4} \mathrm{P}_{1 / 2}$ & 59841.060 & $\left({ }^{3} \mathrm{~F}\right) 5 \mathrm{p}^{2} \mathrm{D}_{3 / 2}^{\circ}$ & 300.7801 & -1.29 & $3.81(7)$ & 10 \\
\hline 27879.033 & $4 d^{5}{ }^{2} F_{5 / 2}$ & 59841.060 & $\left({ }^{3} \mathrm{~F}\right) 5 \mathrm{p}^{2} \mathrm{D}_{3 / 2}^{\circ}$ & 312.7806 & -1.36 & $2.97(7)$ & 12 \\
\hline 32124.340 & $\left({ }^{3} \mathrm{P}\right) 5 \mathrm{~s}{ }^{2} \mathrm{P}_{1 / 2}$ & 59841.060 & $\left({ }^{3} \mathrm{~F}\right) 5 \mathrm{p}^{2} \mathrm{D}_{3 / 2}^{\circ}$ & 360.6902 & -0.77 & $8.55(7)$ & 36 \\
\hline 36289.031 & $\left({ }^{3} \mathrm{~F}\right) 5 \mathrm{~s}^{2} \mathrm{~F}_{5 / 2}$ & 59841.060 & $\left({ }^{3} \mathrm{~F}\right) 5 \mathrm{p}^{2} \mathrm{D}_{3 / 2}^{\circ}$ & 424.4724 & -0.63 & $8.79(7)$ & 61 \\
\hline 39243.688 & ( $\left.{ }^{1} \mathrm{D}\right) 5 \mathrm{~s}^{2} \mathrm{D}_{3 / 2}$ & 59841.060 & $\left({ }^{3} \mathrm{~F}\right) 5 \mathrm{p}^{2} \mathrm{D}_{3 / 2}^{\circ}$ & 485.3634 & -1.17 & $1.89(7)$ & 773 \\
\hline 16947.078 & $4 d^{5}{ }^{4} D_{7 / 2}$ & 60992.764 & $\left({ }^{3} \mathrm{~F}\right) 5 \mathrm{p}^{2} \mathrm{D}_{5 / 2}^{\circ}$ & 226.9668 & -1.37 & $5.55(7)$ & 19 \\
\hline 22444.510 & $4 d^{5}{ }^{2} D_{5 / 2}$ & 60992.764 & $\left({ }^{3} \mathrm{~F}\right) 5 \mathrm{p}^{2} \mathrm{D}_{5 / 2}^{\circ}$ & 259.3376 & -0.87 & $1.34(8)$ & 48 \\
\hline 27410.473 & $4 d^{5}{ }^{2} F_{7 / 2}$ & 60992.764 & $\left({ }^{3} \mathrm{~F}\right) 5 \mathrm{p}^{2} \mathrm{D}_{5 / 2}^{\circ}$ & 297.6891 & -1.08 & $6.31(7)$ & 18 \\
\hline 27627.858 & $\left({ }^{3} \mathrm{P}\right) 5 \mathrm{~s}{ }^{4} \mathrm{P}_{3 / 2}$ & 60992.764 & $\left({ }^{3} \mathrm{~F}\right) 5 \mathrm{p}^{2} \mathrm{D}_{5 / 2}^{\circ}$ & 299.6288 & -1.03 & 7.01(7) & 19 \\
\hline 33146.559 & $\left({ }^{3} G\right) 5 s^{2} G_{7 / 2}$ & 60992.764 & $\left({ }^{3} \mathrm{~F}\right) 5 \mathrm{p}^{2} \mathrm{D}_{5 / 2}^{\circ}$ & 359.0129 & -1.22 & $3.11(7)$ & 50 \\
\hline 34419.517 & $\left({ }^{3} \mathrm{P}\right) 5 \mathrm{~s}{ }^{2} \mathrm{P}_{3 / 2}$ & 60992.764 & $\left({ }^{3} \mathrm{~F}\right) 5 \mathrm{p}^{2} \mathrm{D}_{5 / 2}^{\circ}$ & 376.2114 & -1.17 & $3.13(7)$ & 39 \\
\hline 36741.555 & $\left({ }^{3} \mathrm{~F}\right) 5 \mathrm{~s}^{2} \mathrm{~F}_{7 / 2}$ & 60992.764 & $\left({ }^{3} \mathrm{~F}\right) 5 \mathrm{p}^{2} \mathrm{D}_{5 / 2}^{\circ}$ & 412.2342 & -0.55 & $1.10(8)$ & 66 \\
\hline 37431.722 & $\left({ }^{1} \mathrm{G}\right) 5 \mathrm{~s}^{2} \mathrm{G}_{7 / 2}$ & 60992.764 & $\left({ }^{3} \mathrm{~F}\right) 5 \mathrm{p}^{2} \mathrm{D}_{5 / 2}^{\circ}$ & 424.3099 & -1.19 & $2.38(7)$ & 17 \\
\hline 39913.210 & ( $\left.{ }^{1} \mathrm{D}\right) 5 \mathrm{~s}^{2} \mathrm{D}_{5 / 2}$ & 60992.764 & $\left({ }^{3} \mathrm{~F}\right) 5 \mathrm{p}^{2} \mathrm{D}_{5 / 2}^{\circ}$ & 474.2607 & -1.03 & $2.76(7)$ & 13 \\
\hline
\end{tabular}

${ }^{a}$ Designations and level values from Nilsson and Pickering (2003).

${ }^{\mathrm{b}}$ Experimental results Nilsson (2002). The intensities have not been normalized.

${ }^{c}$ HFR + CPOL calculations: This work.

${ }^{\mathrm{d}}$ Level retained in this table despite of the fact that it has not been measured in the present work (see the text). 
within these two configurations, was also adjusted. For the odd parity, all the experimental levels reported by Nilsson and Pickering (2003) up to $75000 \mathrm{~cm}^{-1}$ were used to optimize the $E_{\mathrm{av}}, F^{k}, G^{k}, \zeta_{n l}, \alpha$ and $\beta$ parameters belonging to $4 \mathrm{~d}^{4} 5 \mathrm{p}$ and the average energy of the $4 d^{3} 5 s 5 p$ configuration. The odd levels above $75000 \mathrm{~cm}^{-1}$ were excluded from the fit because in most cases it was difficult to uniquely relate them to the theoretical values since most of those levels are strongly mixed. The standard deviations of the fits were $84 \mathrm{~cm}^{-1}$ for the even parity (153 levels) and $128 \mathrm{~cm}^{-1}$ for the odd parity (149 levels).

The weighted transition probabilities $(g A)$ and oscillator strengths $(\log g f)$ of the transitions depopulating the levels measured in the present work are reported in table 3 . The table is restricted to the strongest lines $(\log g f>-1.50)$ originating from the 16 odd levels quoted in table 2 , for which lifetimes were measured in the present work. We have added the depopulating channel of the level $\left({ }^{5} \mathrm{D}\right) 5 \mathrm{p} \mathrm{z}{ }^{4} \mathrm{P}_{1 / 2}^{\circ}$ situated at $47208 \mathrm{~cm}^{-1}$, the only level of the $\mathrm{z}^{4} \mathrm{P}^{\circ}$ term not measured in the present work. For comparison the last column of table 3 gives the laboratory intensities obtained by Nilsson (2002) without correcting for the instrumental response.

\section{Results and discussion}

The available lifetimes in Mo II are reported in table 2 where the results obtained in the present work are compared to previous values. When comparing the LIF measurements of Sikström et al (2001) with the lifetimes reported by Hannaford and Lowe (1983), it is seen that the agreement is good when the lifetimes are longer than about 4 ns. For the shorter values, there is a clear discrepancy (outside the error bars), the values of Sikström et al (2001) being shorter. This discrepancy can be explained, as pointed out by Sikström et al (2001), by a better time resolution and shorter excitation pulses in the latter experiment. For the two levels common to our work and that of Sikström et al (2001) there is perfect agreement.

In the last column of table 2, we report the theoretical HFR+CPOL lifetimes calculated by Quinet (2002) (calculation $F$ ) as well as the new results obtained in the present work. The differences between the two calculations are only marginal, the mean ratio being $0.984 \pm 0.030$ for 30 levels (the uncertainty representing the standard deviation around the mean). A very good agreement between the theoretical lifetimes and the experimental results is also observed. In fact, the mean ratio $\tau(\mathrm{HFR}+\mathrm{CPOL}) / \tau(\operatorname{Exp})$ is $0.934 \pm$ 0.100 and $1.011 \pm 0.035$ when considering the measurements of Hannaford and Lowe (1983) and Sikström et al (2001), respectively. The same ratio becomes $1.067 \pm 0.040$ when comparing the 16 experimental lifetimes of the present work with the theoretical HFR+CPOL values. It should also be emphasized that theory favours the shorter lifetimes found by Sikström et al (2001) over those of Hannaford and Lowe (1983).

The importance of core-polarization effects in the Mo II spectrum must not be underestimated. They change the calculated lifetimes by about $30 \%$ as can be seen by comparing calculations $C$ and $F$ in Quinet (2002) and from table 2
Table 4. Oscillator strengths $(\log g f)$ and transition probabilities $(g A)$ in Mo II sorted by air wavelengths. The observed wavelengths and laboratory intensities are taken from Nilsson (2002).

\begin{tabular}{|c|c|c|c|}
\hline$\lambda(\mathrm{nm})$ & $\log g f$ & $g A\left(\mathrm{~s}^{-1}\right)$ & Int. \\
\hline 208.1681 & -1.15 & $1.10(8)$ & 53 \\
\hline 226.9668 & -1.37 & $5.55(7)$ & 19 \\
\hline 230.6981 & -0.44 & $4.55(8)$ & 93 \\
\hline 230.8001 & -1.25 & $7.02(7)$ & 16 \\
\hline 233.2128 & -0.56 & $3.35(8)$ & 82 \\
\hline 233.7424 & -1.29 & $6.25(7)$ & 17 \\
\hline 234.1592 & -0.58 & $3.19(8)$ & 84 \\
\hline 249.8279 & -1.13 & 7.79(7) & 58 \\
\hline 253.8457 & -0.37 & $4.42(8)$ & 300 \\
\hline 254.2672 & -0.84 & $1.50(8)$ & 129 \\
\hline 259.3376 & -0.87 & $1.34(8)$ & 48 \\
\hline 263.8761 & -0.07 & $8.25(8)$ & 948 \\
\hline 267.3270 & -0.29 & $4.84(8)$ & 433 \\
\hline 267.2843 & -0.06 & $8.06(8)$ & 878 \\
\hline 270.3612 & -1.21 & $5.64(7)$ & 19 \\
\hline 271.7348 & -0.37 & $3.87(8)$ & 376 \\
\hline 271.3504 & -0.82 & $1.38(8)$ & 119 \\
\hline 273.6956 & -0.71 & $1.74(8)$ & 169 \\
\hline 274.6297 & -0.48 & $2.94(8)$ & 271 \\
\hline 276.3616 & -0.61 & $2.18(8)$ & 182 \\
\hline 277.3780 & -1.19 & $5.58(7)$ & 43 \\
\hline 277.4392 & -0.78 & $1.43(8)$ & 122 \\
\hline 278.4987 & -0.19 & $5.59(8)$ & 327 \\
\hline 280.2353 & -1.33 & $4.05(7)$ & 27 \\
\hline 280.7753 & -0.43 & $3.13(8)$ & 222 \\
\hline 281.7441 & -1.11 & $6.50(7)$ & 39 \\
\hline 283.4396 & -0.90 & $1.05(8)$ & 47 \\
\hline 284.2148 & -0.92 & $9.86(7)$ & 61 \\
\hline 286.6688 & -0.49 & $2.66(8)$ & 84 \\
\hline 289.1273 & -0.73 & $1.49(8)$ & 33 \\
\hline 289.2808 & -0.66 & $1.75(8)$ & 48 \\
\hline 290.7112 & -0.96 & $8.82(7)$ & 74 \\
\hline 292.3220 & -1.13 & $5.83(7)$ & 43 \\
\hline 297.6891 & -1.08 & $6.31(7)$ & 18 \\
\hline 299.6288 & -1.03 & 7.01(7) & 19 \\
\hline 300.7801 & -1.29 & $3.81(7)$ & 10 \\
\hline 305.2322 & -0.82 & $1.08(8)$ & 17 \\
\hline 308.7621 & 0.43 & $1.89(9)$ & 213 \\
\hline 309.7687 & -0.81 & $1.07(8)$ & 24 \\
\hline 309.2074 & -0.75 & $1.25(8)$ & 212 \\
\hline 311.1636 & -1.13 & $5.06(7)$ & 21 \\
\hline 312.1995 & 0.32 & $1.43(9)$ & 216 \\
\hline 312.7806 & -1.36 & $2.97(7)$ & 12 \\
\hline 313.6466 & -0.46 & $2.35(8)$ & 159 \\
\hline 313.8477 & -1.10 & $5.37(7)$ & 31 \\
\hline 313.8717 & -0.14 & $4.90(8)$ & 82 \\
\hline 314.4625 & -1.29 & $3.46(7)$ & 10 \\
\hline 315.2820 & 0.18 & $1.03(9)$ & 197 \\
\hline 315.8932 & -1.03 & $6.17(7)$ & 8 \\
\hline 317.5051 & -0.32 & $3.20(8)$ & 89 \\
\hline 317.8004 & -0.94 & 7.67(7) & 12 \\
\hline 317.2744 & -0.90 & $8.28(7)$ & 169 \\
\hline 318.3405 & -1.04 & $5.98(7)$ & 11 \\
\hline 318.6382 & -1.22 & $3.95(7)$ & 51 \\
\hline 318.7587 & -0.62 & $1.56(8)$ & 136 \\
\hline 319.5847 & -1.22 & $3.92(7)$ & 30 \\
\hline 319.2097 & -1.25 & $3.67(7)$ & 83 \\
\hline 320.1499 & -1.34 & $2.97(7)$ & 57 \\
\hline 324.0715 & -0.95 & 7.13(7) & 152 \\
\hline 325.0738 & -0.79 & $1.01(8)$ & 107 \\
\hline 325.8677 & -1.40 & $2.49(7)$ & 55 \\
\hline 326.7633 & -0.81 & $9.73(7)$ & 77 \\
\hline 327.1662 & -0.64 & $1.41(8)$ & 135 \\
\hline
\end{tabular}


Table 4. (Continued.)

\begin{tabular}{lrlr}
\hline$\lambda(\mathrm{nm})$ & $\log g f$ & $g A\left(\mathrm{~s}^{-1}\right)$ & Int. \\
\hline 328.7200 & -1.38 & $2.56(7)$ & 57 \\
329.0704 & -0.74 & $1.12(8)$ & 127 \\
329.2313 & 0.18 & $9.28(8)$ & 765 \\
329.7683 & -0.81 & $9.56(7)$ & 97 \\
332.0902 & -0.23 & $3.58(8)$ & 329 \\
332.1189 & -1.20 & $3.79(7)$ & 46 \\
332.9212 & -0.46 & $2.09(8)$ & 233 \\
334.6396 & -0.43 & $2.19(8)$ & 206 \\
334.7267 & -0.70 & $1.17(8)$ & 137 \\
336.2998 & -0.70 & $1.19(8)$ & 23 \\
337.9755 & -0.68 & $1.21(8)$ & 151 \\
338.0213 & -0.57 & $1.55(8)$ & 165 \\
343.5376 & -0.43 & $2.06(8)$ & 35 \\
344.5500 & -0.63 & $1.33(8)$ & 38 \\
344.6083 & -0.54 & $1.63(8)$ & 176 \\
350.1942 & -0.72 & $1.04(8)$ & 25 \\
352.4587 & -0.73 & $9.95(7)$ & 169 \\
353.4686 & -1.30 & $2.70(7)$ & 74 \\
354.5990 & -1.00 & $5.35(7)$ & 14 \\
354.7942 & -1.00 & $5.26(7)$ & 15 \\
355.9713 & -1.38 & $2.20(7)$ & 22 \\
359.0129 & -1.22 & $3.11(7)$ & 50 \\
359.6347 & -0.97 & $5.56(7)$ & 71 \\
360.6902 & -0.77 & $8.55(7)$ & 36 \\
362.3685 & -1.19 & $3.27(7)$ & 26 \\
363.5143 & 0.04 & $5.56(8)$ & 579 \\
367.0666 & -1.11 & $3.90(7)$ & 49 \\
368.8303 & -0.18 & $3.30(8)$ & 479 \\
369.2640 & -0.24 & $2.85(8)$ & 446 \\
370.2546 & -0.44 & $1.79(8)$ & 262 \\
371.9777 & -1.13 & $3.60(7)$ & 57 \\
374.2338 & -0.89 & $6.23(7)$ & 93 \\
374.6433 & -1.13 & $3.53(7)$ & 25 \\
374.8113 & -1.07 & $4.05(7)$ & 38 \\
375.5489 & -0.91 & $5.88(7)$ & 94 \\
376.2114 & -1.17 & $3.13(7)$ & 39 \\
386.1289 & -1.06 & $3.91(7)$ & 67 \\
396.8651 & -1.32 & $2.03(7)$ & 59 \\
412.2342 & -0.55 & $1.10(8)$ & 66 \\
424.3099 & -1.19 & $2.38(7)$ & 17 \\
424.4724 & -0.63 & $8.79(7)$ & 61 \\
427.9011 & -1.00 & $3.67(7)$ & 117 \\
436.3632 & -0.96 & $3.87(7)$ & 119 \\
437.7758 & -1.08 & $2.90(7)$ & 93 \\
443.3496 & -1.14 & $2.47(7)$ & 85 \\
474.2607 & -1.03 & $2.76(7)$ & 13 \\
485.3634 & -1.17 & $1.89(7)$ & 773 \\
\hline & & &
\end{tabular}

in Nilsson and Pickering (2003). Their inclusion in the calculations is thus essential for obtaining an accurate scale for the transition probabilities.

Weighted transition probabilities $(g A)$ and oscillator strengths $(\log g f)$ are reported in table 3 for $110 \mathrm{UV}$ transitions $(208<\lambda<490 \mathrm{~nm})$. They have been obtained by a combination of the experimental lifetimes and the theoretical
BFs. No experimental BFs are available for these highexcitation levels. There are no other results available for comparison, but the absolute scale is well established on the basis of the available lifetimes as discussed above. For the convenience of the user, table 4 lists the oscillator strengths and transition probabilities sorted by air wavelengths.

The new results for Mo II reported in this paper fill in some gaps in the existing data for this ion of astrophysical interest and it is anticipated that they will help astrophysicists in quantitative investigations of stellar spectra, particularly for investigating the chemical composition of Ap stars.

\section{Acknowledgments}

This work was financially supported by the Integrated Initiative of Infrastructure Project LASERLAB-EUROPE, contract RII3-CT-2003-506350, the Swedish Research Council through the Linnaeus grant, the Knut and Alice Wallenberg Foundation and the Belgian FRS-FNRS. EB, PQ and PP are, respectively, Research Director, Senior Research Associate and Research Associate of the FRS-FNRS. They are greatly indebted to the Swedish team for the warm hospitality enjoyed at the Lund Laser Center during the measurements.

\section{References}

Bergström H, Faris H, Hallstadius G W, Lundberg H, Persson A and Wahlström C G 1988 Z. Phys. D 817

Biémont É, Palmeri P, Quinet P, Paquin G, Zhang Z G, Somesfalean G and Svanberg S 2001 Mon. Not. R. Astron. Soc. 3281085

Corliss C H and Bozman W R 1962 Natl Bur. Stand. Monogr. vol 53 (Washington, DC: US Govt Printing Office)

Cowan R D 1981 The Theory of Atomic Structure and Spectra (Berkeley, CA: University of California Press)

Fraga S, Karwowski J and Saxena K M S 1976 Handbook of Atomic Data (Amsterdam: Elsevier)

Hannaford P and Lowe R M 1983 J. Phys. B: At. Mol. Phys. 164539

Kiess C C 1958 J. Res. Natl Bur. Stand. 60375

Kwiatkowski M, Micali G, Werner K and Zimmermann P 1981 Phys. Lett. 85A 273

Meggers W F and Kiess C C 1926 J. Opt. Soc. Am. 12417

Nilsson H 2002 PhD Thesis University of Lund, Sweden

Nilsson H and Pickering J C 2003 Phys. Scr. 67223

Quinet P 2002 J. Phys. B: At. Mol. Opt. Phys. 3519

Rau V R 1949 Indian J. Phys. 23387

Schauls R and Sawyer R A 1940 Phys. Rev. 58781

Schnehage S E, Danzmann K, Kuennemeyer R and Kock M 1983 J. Quant. Spectrosc. Radiat. Transfer 29507

Sikström C M, Pihlemark H, Nilsson H, Litzén U, Johansson S, Li Z S and Lundberg H 2001 J. Phys. B: At. Mol. Opt. Phys. 34477

Sugar J and Musgrove A 1988 J. Phys. Chem. Ref. Data 17155

Xu H L, Persson A, Svanberg S, Blagoev K, Malcheva G, Pentchev V, Biémont É, Campos J, Ortiz M and Mayo R 2004 Phys. Rev. A 70042508

Zhang Z G, Li Z S, Svanberg S, Palmeri P, Quinet P and Biémont É 2001 Eur. Phys. J. D 15301 